\title{
Efecto del manejo silvicultural sobre la regeneración de un bosque de Nothofagus dombeyi, N. alpina y N. obliqua en la Reserva Nacional Lanín (Argentina)
}

\author{
Effect of silvicultural management on the Nothofagus dombeyi, N. alpina and N. obliqua forest \\ regeneration within the Lanín Natural Reserve (Argentina)
}

\author{
Georgina Sola a,b,c*, Hernán Attis Beltrán ${ }^{\text {a,c }}$, Luis Chauchard ${ }^{\text {c,d }}$, Leonardo Gallo ${ }^{\text {b }}$ \\ ${ }^{a}$ Consejo Nacional de Investigaciones Científicas y Técnicas (CONICET), Argentina.

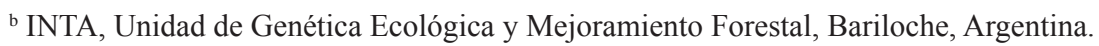 \\ *Autor de correspondencia: ' Universidad Nacional del Comahue, Cátedra de Ordenación Forestal, Pasaje de La Paz 235, \\ San Martín de Los Andes, Neuquén, Argentina, tel.: 54-02972-427618, solageor@yahoo.com.ar. \\ d Administración de Parques Nacionales, Parque Nacional Lanín, Neuquén, Argentina.
}

\begin{abstract}
SUMMARY
Management practices are important disturbance agents at local level and their incorrect implementation may affect the sustainability of natural forests. Usually, silviculture based on the shelterwood system was carried out in the mixed stands composed of Nothofagus dombeyi, N. alpina and N. obliqua, located at Lanín Natural Reserve (Neuquén Province, Argentina). However, the effect of this scheme on the composition and structure of natural regeneration is still rather unknown. In this study, composition, age, abundance and spatial pattern of adults and saplings of Nothofagus species were evaluated in a 3-ha plot logged in 1993. At pre-harvest period, adult density was $26 \%$ for $N$. dombeyi, $38 \%$ for $N$. alpina and $35 \%$ for $N$. obliqua. Regeneration consisted mainly of $N$. nervosa. After logging, composition of adult trees was $20 \%, 43 \%$ and $37 \%$ for $N$. dombeyi, $N$. alpina, $N$. obliqua, respectively; whereas regeneration was $45 \%$ for $N$. obliqua and $42 \%$ for $N$. dombeyi. Within species, no relationship was found between basal area of adult trees and density of saplings. For all tree species, age regeneration was larger in areas where tree canopy cover exhibited higher values. $N$. alpina was the last installed species. Preservation of the relative species composition after logging did not maintain the original abundance within regeneration. Consequently, it is necessary to adjust silvicultural practices, and particularly the level of canopy openings, in order to maintain species composition unaffected as a key indicator of sustainability at management unit scale.
\end{abstract}

Key words: silvicultural management, basal area, tree canopy, Lophosonia.

\section{RESUMEN}

Las prácticas silviculturales constituyen un importante agente de disturbio y su inadecuada implementación afecta la sostenibilidad de los bosques naturales. En la Reserva Nacional Lanín, el manejo silvicultural del bosque de Nothofagus dombeyi, N. alpina y N. obliqua se realiza tradicionalmente mediante aclareos sucesivos; y se desconoce su efecto sobre la composición y estructura de la regeneración natural. La cobertura del dosel, composición y área basal de los individuos adultos, y la composición, edad y distribución espacial de los renovales fue evaluada en una parcela de 3 ha intervenida en 1993 mediante una corta diseminatoria. La densidad de adultos antes de la corta fue $26 \%$ para $N$. dombeyi, $38 \%$ para $N$. alpina y $35 \%$ para $N$. obliqua, y la remanente fue 20 , 44 y $36 \%$, respectivamente. Previamente al aprovechamiento, el $92 \%$ de la regeneración estaba constituida por N. alpina, y luego principalmente por $N$. obliqua (45\%) y $N$. dombeyi (42\%). Estos valores no se correlacionaron con el área basal de adultos. La edad de los renovales fue mayor en sitios con alta cobertura de dosel y N. alpina fue la especie que se instaló última. El aprovechamiento del bosque de Nothofagus no promovió el mantenimiento de la composición relativa original de la regeneración. El tiempo de colonización de las especies de Nothofagus disminuiría y una mayor cantidad de renovales de $N$. alpina, especie más tolerante a la sombra, se establecería si la cobertura remanente fuera superior a la recomendada en los planes de manejo.

Palabras clave: aclareos sucesivos, cobertura del dosel, área basal, Lophozonia.

\section{INTRODUCCIÓN}

Las prácticas silviculturales constituyen un importante agente de disturbio y su inadecuada implementación afecta la sostenibilidad de los bosques naturales.
En los ecosistemas forestales, el disturbio es un componente clave que determina la composición y abundancia de las especies arbóreas. La escala, intensidad y el patrón espacial del disturbio define la composición de la vegetación subsecuente (Pollmann 2002). En el 
manejo del bosque, el cambio en la abundancia relativa de las especies podría ocasionar modificaciones en la población efectiva que da origen a la regeneración (Barik et al. 1996). Es decir, el aprovechamiento silvicultural modifica la estructura forestal que puede implicar un impacto significativo sobre las variables bióticas y abióticas del bosque, y por ende sobre la composición de la regeneración (Martínez Pastur et al. 2002). Por lo tanto, el manejo forestal se debe llevar a cabo desde una perspectiva integral, manteniendo la variabilidad natural de los ecosistemas afectados. La silvicultura debe ser compatible con las estrategias de regeneración de las especies teniendo en cuenta el régimen de disturbios naturales para poder predecir y reducir los impactos en el ecosistema (Smith et al. 1997).

La degradación del bosque puede ser causada por la extracción selectiva positiva, que consiste en la remoción de los árboles con mayor valor comercial y el mantenimiento de una masa residual compuesta por individuos de baja calidad maderera. Esto puede producir además un impacto en la estructura genética por una selección disgénica, con la consecuente disminución de la aptitud productiva del bosque (Finkeldey y Ziehe 2004). Esta práctica implica beneficios económicos a corto plazo pero no considera el impacto sobre la biodiversidad, la regeneración y el desarrollo del bosque (Donoso 2013).

Las principales variables que influyen sobre el establecimiento y crecimiento de los renovales son la disponibilidad de luz y humedad en el suelo, y la intervención silvicultural influye sobre estas variables debido principalmente a los cambios en la cobertura del dosel (Dezzotti et al. 2003, Promis et al. 2010). El cambio generado por la intervención forestal sobre el microclima del bosque puede generar efectos negativos en la respuesta de las diferentes especies.

En la Reserva Nacional Lanín ( $40^{\circ} 9^{\prime} \mathrm{S}$ y $\left.71^{\circ} 21^{\prime} \mathrm{O}\right)$, desde fines de la década de 1980 se realiza el manejo silvicultural del bosque de Nothofagus dombeyi (Mirb.) Oerst. (coihue), Nothofagus alpina (Poepp. et Endl.) Oerst. (raulí) y Nothofagus obliqua (Mirb.) Oerst. (roble pellín) (Nothofagaceae). En la Argentina, este tipo de vegetación ocupa 313.000 ha y se localiza entre los $39^{\circ} 29^{\prime}$ y $40^{\circ} 22^{\prime} \mathrm{S}$ y los $71^{\circ} 15^{\prime}$ y $71^{\circ} 40^{\prime} \mathrm{O}$ (Lara et al. 1999). El sistema silvicultural principalmente aplicado a este bosque mixto es el aclareo sucesivo, que a lo largo de una serie de cortas disminuye la cobertura del dosel hasta un 30 ó $40 \%$, y mantiene los árboles adultos remanentes homogéneamente distribuidos y con una composición relativa similar a la del rodal original ${ }^{1}$. El establecimiento de la regeneración se extiende aproximadamente 20 años durante el cual se realizan usualmente, dos cortas diseminatorias y una de liberación (Chauchard et al. 2001).

Chauchard L, M González Peñalba y L Maresca. 1998. Plan de ordenación forestal "Chachin", Reserva Nacional Lanín, Primera revisión ordinaria. Administración de Parques Nacionales. No publicado.
Los renovales de estas especies de Nothofagus recolonizan los sitios abiertos cuando la vegetación original es eliminada a través de disturbios periódicos y de gran escala (Veblen 1996). Se han observado diferencias entre las especies con relación a la sobrevivencia y el crecimiento de los renovales bajo diferentes regímenes de disturbios menores que afectan, entre otros factores ambientales, a la luminosidad (Read y Hill 1985, Grosse 1988, Ramírez et al. 1997, Weinberger y Ramírez 1999, Pollmann 2002, Pollmann y Veblen 2004). Las especies que componen el bosque mixto tienen diferentes respuestas a estos cambios; $N$. alpina es una especie semitolerante a la sombra mientras que $N$. dombeyi y $N$. obliqua demandan una intensidad de luz mayor (Weinberger y Ramírez 2001).

El objetivo del presente estudio es evaluar la densidad y estructura espacial de los renovales de $N$. dombeyi, $N$. alpina y $N$. obliqua y su relación con el estrato de árboles adultos luego de 20 años de la implementación del aclareo sucesivo, para contribuir a diseñar esquemas de manejo que promuevan la regeneración, mantengan la composición original y disminuyan el tiempo de establecimiento de estas especies.

\section{MÉTODOS}

El área de estudio se localizó en la ladera Sur del cerro Quilanlahue ( $40^{\circ} 8^{\prime} \mathrm{S}$ y $\left.71^{\circ} 28^{\prime} \mathrm{O}\right)$, en jurisdicción de la Reserva Nacional Lanín (provincia de Neuquén, Argentina) (figura 1). El clima es templado húmedo (temperatura media anual $9{ }^{\circ} \mathrm{C}$ y precipitación total anual $2.360 \mathrm{~mm}$ ) y los vientos predominantes son del sector Oeste. El relieve es montañoso y los suelos de origen volcánico se clasifican como Andisoles (Ferrer et al. 1991).

En un bosque mixto compuesto por N. dombeyi, N. alpina y $N$. obliqua ubicado a $900 \mathrm{~m}$ s.n.m. se estableció una parcela de tres hectáreas. En 1993 se implementó una corta diseminatoria dentro de un esquema de aclareos sucesivos, y se alcanzó la meta de 30 a $40 \%$ de cobertura del dosel por lo que no se realizó una segunda corta. La composición, el área basal y la cobertura del dosel superior del rodal previo a la implementación de la corta se obtuvieron del inventario realizado para elaborar el plan de manejo ${ }^{2}$.

En el 2013, a todos los árboles adultos (diámetro a la altura del pecho, DAP $\geq 10 \mathrm{~cm}$ ) presentes en una parcela de 3 ha se les midió el DAP $(\mathrm{cm})$ (cinta diamétrica) y la altura total $(\mathrm{Ht}, \mathrm{m})$ (clinómetro y cinta métrica). Posteriormente, se seleccionaron 20 individuos sanos y no excéntricos de cada especie que representaron el rango de diámetros presentes en la parcela, y se les extrajeron tarugos a la altura del pecho (barreno de Pressler). A estos individuos se les estimó la edad a través de la cuenta de los anillos de crecimiento (lupa 10X), a la cual se le sumó el tiempo que

Chauchard L. 1989. Plan de ordenación Quilanlahue. Municipalidad de Junín de los Andes-Administración de Parques Nacionales. No publicado. 


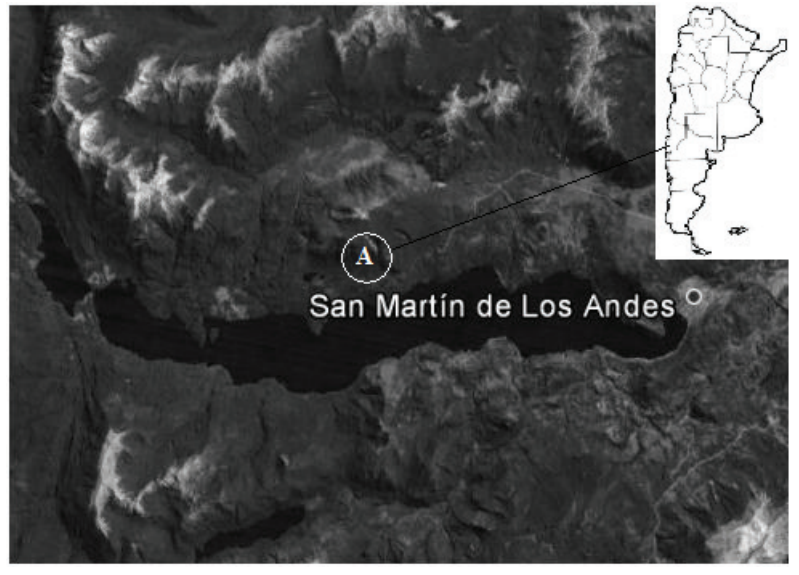

Figura 1. A: Área de estudio. Quilanlahue, Reserva Nacional Lanín, Neuquén, Argentina.

Study area. Quilanlahue, Reserva Nacional Lanín, Neuquén, Argentina.

tardaron en alcanzar 1,3 $\mathrm{m}$ de altura y que se obtuvo de la función desarrollada para estimar la edad de los renovales (DAP $<10 \mathrm{~cm}$ ) (cuadro 3).

A lo largo de 14 transectas ubicadas sistemáticamente cada $20 \mathrm{~m}$ se instalaron 80 subparcelas de $4 \mathrm{~m}^{2}$ cada una, en las cuales se midió la altura (cinta métrica) y el diámetro (calibre) a la base de todos los renovales presentes. La comparación de la densidad de renovales por especie se realizó mediante la prueba de Kruskal-Wallis ya que los datos no pudieron ser normalizados. La edad de los renovales (E, años) se estimó a partir de la medición de la Ht y el conteo de anillos a la altura del cuello (lupa 10x) de 60 individuos de cada especie que abarcaron el rango de $\mathrm{Ht}$.

El tipo y la intensidad del patrón espacial de los renovales se estimó a través del Índice de Morisita (Morisita 1959), de acuerdo a:

$$
\mathrm{I}_{\delta}=\mathrm{q}\left(\sum \mathrm{n}_{\mathrm{i}}\left(\mathrm{n}_{\mathrm{i}}-1\right)\right) / \mathrm{N}(\mathrm{N}-1)
$$

Donde: $I_{\delta}$ es el índice de Morisita, q es la cantidad de subparcelas en la que se dividió la parcela, $\mathrm{n}_{\mathrm{i}}$ es la cantidad de individuos en la i-ésima subparcela y $\mathrm{N}$ es la cantidad total de individuos $\left(=\Sigma \mathrm{n}_{\mathrm{i}}\right)$. El índice $\mathrm{I}_{\delta}$ es igual a 1 cuando los individuos se dispersan aleatoriamente, es mayor a 1 cuando el patrón espacial es agrupado y es menor a 1 cuando es regular.

Luego se utilizó una prueba de Chi-cuadrado $\left(X^{2}\right)$ para determinar si el $\mathrm{I}_{\delta}$ calculado fue estadísticamente diferente a 1 , de acuerdo a:

$$
X_{\text {calculado }}^{2}=\mathrm{I}_{\delta}(\mathrm{N}-1)+\mathrm{q}-\mathrm{N}
$$

Si $X_{\text {calculado }}^{2}>X_{(\mathrm{q}-1 ; 0,05)}^{2}$ el patrón es diferente al aleatorio y si $X_{\text {calculado }}^{2} \leq X_{(\mathrm{q}-1 ; 0,05)}^{2}$ el patrón es aleatorio.

Para analizar si la densidad y proporción de especies de la regeneración dependían de los individuos adultos que la rodeaban, se relacionó dicha densidad con el área basal de los árboles adultos circundantes. Se utilizó el área basal porque está directa y fuertemente relacionada con el volumen y el tamaño de la copa (Chauchard y Sbrancia 2003). La misma se determinó por especie, en un radio de $25 \mathrm{~m}$ desde el centro de las subparcelas. Se utilizó esta distancia ya que la dispersión de semillas en los Nothofagus se ve limitada a la cercanía de los árboles padre (Veblen et al. 1996). Mediante un análisis de correspondencia canónica se analizó la relación entre el área basal por especie de los árboles circundantes y la cantidad de renovales de esa especie presentes en las subparcelas.

La influencia de dosel superior remanente sobre el establecimiento de la regeneración se evaluó a partir de la medición de la cobertura de copas, a través de fotografías hemisféricas tomadas en enero 2013 en el centro de cada subparcela a $1,2 \mathrm{~m}$ sobre el nivel del suelo y en días nublados para optimizar el contraste entre el follaje y el cielo. El equipo consistió en una cámara Nikon D80 provista de una lente hemisférica SIGMA 4,5 mm, montada sobre un trípode para mantener la lente en forma horizontal, con el cual se tomaron fotografías verticales cuyo norte magnético quedó en su parte superior. La apertura de la canopia se estimó con el programa Gap Light Analyzer 2.0 (Frazer et al. 2000). Estos valores se convirtieron a cobertura, la cual se clasificó en baja o alta en base al rango de valores actuales y el punto de corte fue el valor medio. Además, en cada subparcela se registró la pendiente, la exposición, el cénit, la altitud y las coordenadas geográficas (GPS). Con dicha información se realizaron análisis de la varianza (ANDEVA) multifactoriales para establecer si la edad de las especies difería con la cobertura y con el área basal de los individuos adultos circundantes de la misma especie. Para dicho análisis el área basal se clasificó en baja o alta, en función del rango de datos y teniendo en cuenta los valores utilizados en los planes de manejo. Finalmente, se elaboró un gráfico para cada cobertura con la densidad de renovales establecidos por especie divididos en dos clases ( 0 - 5 años y $5-10$ años posteriores a la corta diseminatoria). Se consideraron renovales establecidos a aquellos que superaban la altura del sotobosque ${ }^{3}$.

\section{RESULTADOS}

Previamente al aprovechamiento silvicultural, la densidad de adultos de $N$. dombeyi fue $26 \%$, la de $N$. alpina $38 \%$ y la de $N$. obliqua $35 \%$, $N$. alpina presentó la mayor área basal (cuadro 1). La cobertura promedio fue $85 \%{ }^{4}$. Posteriormente, la densidad de N. alpina fue $44 \%$, mien-

\footnotetext{
Ver nota 2.

4 Ver nota 2.
} 
Cuadro 1. Parámetros estructurales de los árboles adultos del bosque mixto de Nothofagus.

Characterization of the forest structure.

\begin{tabular}{|c|c|c|c|c|c|c|c|c|}
\hline \multirow[b]{2}{*}{ Especie } & \multicolumn{2}{|c|}{ Pre-aprovechamiento } & \multicolumn{6}{|c|}{ Post-aprovechamiento } \\
\hline & $\begin{array}{c}\text { Densidad } \\
\left.\text { árboles ha-1 }{ }^{-1} \%\right)\end{array}$ & $\begin{array}{l}\text { Área basal } \\
\mathrm{m}^{2} \mathrm{ha}^{-1}(\%)\end{array}$ & $\mathrm{n}$ & $\begin{array}{c}\text { Densidad } \\
\text { árboles ha-1 }(\%)\end{array}$ & $\begin{array}{l}\text { Área basal } \\
\mathrm{m}^{2} \text { ha }^{-1}(\%)\end{array}$ & $\begin{array}{l}\text { Altura (m) } \\
\bar{X} \pm E E\end{array}$ & $\begin{array}{l}\text { Diámetro } \\
\text { (cm) } \bar{X} \pm \mathrm{EE}\end{array}$ & $\begin{array}{c}\text { Edad (años) } \\
\bar{X} \pm E E\end{array}$ \\
\hline N. dombeyi & $45(26)$ & $10(22)$ & 51 & $17(20)$ & $11(30)$ & $27 \pm 9$ & $81 \pm 56$ & $178 \pm 96$ \\
\hline N. alpina & $65(38)$ & $22(46)$ & 113 & $38(44)$ & $11(30)$ & $23 \pm 8$ & $53 \pm 32$ & $136 \pm 86$ \\
\hline N. obliqua & $60(35)$ & $16(32)$ & 95 & $32(36)$ & $15(40)$ & $31 \pm 7$ & $74 \pm 22$ & $187 \pm 81$ \\
\hline Total & $170(100)$ & $48(100)$ & 259 & $86(100)$ & $37(100)$ & - & - & - \\
\hline Promedio & - & - & - & - & - & $27 \pm 9$ & $66 \pm 37$ & $167 \pm 89$ \\
\hline
\end{tabular}

tras que la de $N$. obliqua se mantuvo en $36 \%$ y la de $N$. dombeyi disminuyó a $20 \%$ (cuadro 1). Sin embargo, la participación en área basal fue homogénea, con una leve supremacía de $N$. obliqua. El $92 \%$ los individuos con DAP entre 10 y $20 \mathrm{~cm}$ correspondieron a N. alpina y sólo el $2 \%$ a $N$. obliqua, cuya edad promedio fue $54 \pm 20$ años y no existieron individuos con menos de 20 años (cuadro 1).

Los renovales se localizaron en el $65 \%$ de las subparcelas, exhibieron un índice de Morisita de 9,9 para N. dombe$y i, 2,8$ para $N$. alpina y 3,3 para $N$. obliqua y estos valores difirieron significativamente de 1 (prueba de $X^{2}, P \leq 0,05$ ). La densidad relativa de renovales de $N$. dombeyi (44\%) respecto de $N$. alpina (17\%) fue inversa a la de los adultos, mientras que la de $N$. obliqua se mantuvo semejante (cuadro 2, figura 2). El área basal de los árboles adultos circundantes a las parcelas y la cantidad de renovales por especie no estuvo correlacionado (Análisis de correspondencias canónicas, $P>0,05$ ).

El modelo para estimar la edad en función de la altura obtuvo estadísticos de ajustes satisfactorios y sus residuales no mostraron tendencias en su dispersión (cuadro 3). $N$. alpina, a pesar de ser la especie dominante en el dosel superior, presentó un menor número de renovales (prueba de Kruskal-Wallis, $P=0,0014$ ), siendo estos los más jóvenes (cuadro 4, figura 3 ).

El establecimiento de la regeneración difirió entre sitios con bajas y altas coberturas y entre las especies de Nothofagus (cuadro 4, figura 3). Las tres especies tendieron a instalarse antes en los sitios con mayores coberturas, mientras que en aquellos con coberturas menores a $68 \%$ el reclutamiento más tardío fue el de $N$. alpina. El establecimiento de $N$. dombeyi se produjo antes en los sitios donde el área basal de los árboles adultos circundantes fue mayor (cuadro 5, figura 4). Además, la densidad de los renovales establecidos que corresponden a aquellos que superaron la altura del sotobosque, fue superior en sitios con alta cobertura. El establecimiento de la regeneración comenzó luego de 5 años de realizadas las cortas donde la cobertura del dosel fue menor, mientras que en el resto se inició casi inmediatamente (figura 5).

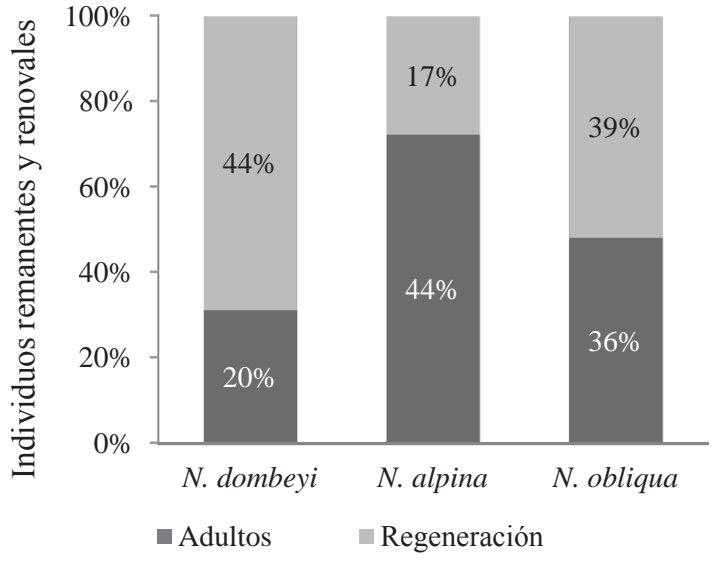

Figura 2. Proporción de individuos adultos remanentes y renovales de las especies de Nothofagus (\%).

Adults and regeneration frequencies (\%).

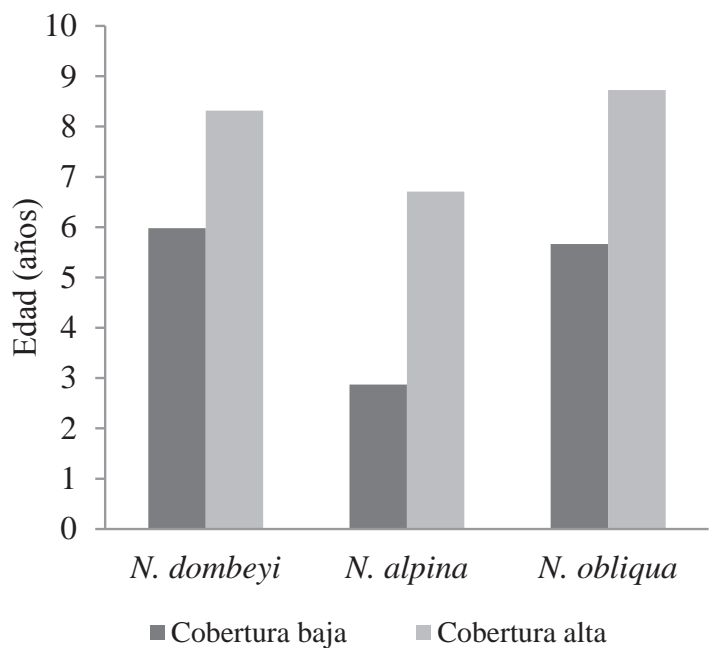

Figura 3. Edad promedio por especie para dos niveles de cobertura (ANDEVA multifactorial, $p<0,05, \mathrm{n}=408$ ).

Average age for two levels of canopy tree (Multiple-way ANOVA, $P<0.05 ; \mathrm{n}=407$ ). 
Cuadro 2. Parámetros de la regeneración posterior al aprovechamiento silvicultural. $\bar{X}$ : media, EE: error estándar.

Characterization of post-harvest regeneration.

\begin{tabular}{lccccc}
\hline \multicolumn{1}{c}{ Especie } & $\begin{array}{c}\text { Densidad } \\
\left(\text { árboles ha } \mathrm{a}^{-1}\right)(\%)\end{array}$ & $\begin{array}{c}\text { Altura }(\mathrm{m}) \\
\overline{\mathrm{X}} \pm \mathrm{EE}\end{array}$ & $\begin{array}{c}\text { Diámetro }(\mathrm{cm}) \\
\overline{\mathrm{X}} \pm \mathrm{EE}\end{array}$ & $\begin{array}{c}\text { Edad (años) } \\
\overline{\mathrm{X}} \pm \mathrm{EE}\end{array}$ & $\mathrm{n}$ \\
\hline N. dombeyi & $5.593,75(44)$ & $1,74 \pm 1,25$ & $1,77 \pm 1,76$ & $7,13 \pm 3,20$ & 179 \\
N. alpina & $2.218,75(17)$ & $0,66 \pm 1,22$ & $0,50 \pm 1,09$ & $3,57 \pm 3,36$ & 71 \\
N. obliqua & $4.938,00(39)$ & $1,65 \pm 1,53$ & $1,32 \pm 1,54$ & $6,46 \pm 3,61$ & 158 \\
Total & $12.781,25(100)$ & - & - & - & 408 \\
Promedio & - & $1,52 \pm 1,42$ & $1,77 \pm 1,64$ & $6,25 \pm 3,61$ & - \\
\hline
\end{tabular}

Cuadro 3. Parámetros del modelo lineal $(\mathrm{E}=\mathrm{a}+\mathrm{b} \mathrm{Ht})$ para los renovales de las especies de Nothofagus. Se indica el coeficiente de determinación $\left(\mathrm{R}^{2}\right)$, el error estándar de la media (EE) y el tamaño de la muestra (n).

Age-height model for the three species.

\begin{tabular}{lccccc}
\hline \multicolumn{1}{c}{ Especie } & $\mathrm{a}$ & $\mathrm{b}$ & $\mathrm{R}^{2}$ & $\mathrm{EE}$ & $\mathrm{n}$ \\
\hline N. dombeyi & 4,46 & 1,73 & 0,64 & 1,21 & 60 \\
N. alpina & 3,70 & 1,64 & 0,61 & 1,44 & 60 \\
N. obliqua & 3,78 & 1,64 & 0,58 & 1,20 & 60 \\
\hline
\end{tabular}

Cuadro 4. ANDEVA multifactorial para la edad de los renovales de Nothofagus en función de la cobertura remanente (Baja: 55 - $68 \%$ y Alta: 68 - $80 \%$ ). Letras distintas indican diferencias significativas (prueba de Bonferroni, $P<0,05, \mathrm{n}=408$ ). Nivel de significancia: $*=P<0,05, * *=P<0,01, * * *=P<0,001$ y $\mathrm{ns}=$ no significativo.

Multiple factor ANOVAs performed on saplings age $(n=408)$ considering after logging tree cover (low: 55-68 \% and high: 68-80\%) and species as main factors.

\begin{tabular}{ccc}
\hline Factor & Nivel & Edad (años) \\
\hline \multirow{2}{*}{ A: Cobertura } & Baja & $4,84 \mathrm{a}$ \\
\cline { 2 - 3 } & Alta & $7,92 \mathrm{~b}$ \\
\hline B: Especie & N. dombeyi & $74,79(* * *)$ \\
& N. alpina & $4,79 \mathrm{~b}$ \\
& $N$. obliqua & $7,19 \mathrm{a}$ \\
\hline A x B & $\mathrm{F}(*)$ & $9,97(* * *)$ \\
\hline
\end{tabular}

Cuadro 5. ANDEVA multifactorial para la edad de los renovales de Nothofagus con relación a la cobertura remanente (Baja: 5568 \% y Alta: 68-80\%) y el área basal de los individuos adultos circundantes (Baja: 0-15 $\mathrm{m}^{2} /$ ha y Alta: $>15 \mathrm{~m}^{2} / \mathrm{ha}$ ). Letras distintas indican diferencias significativas (prueba de Bonferroni, $P<0,05)$. Nivel de significancia: $*=P<0,05 ; * *=P<0,01 ; * * *$ $=P<0,001 ; \mathrm{ns}=$ no significativo.

Multiple factor ANOVAs performed on saplings age considering after logging tree canopy (low: 55-68 \% and high: 68-80\%) and basal area of surrounding adult trees (low: 0-15 $\mathrm{m}^{2}$ ha $^{-1}$ and high: $>15 \mathrm{~m}^{2}$ ha $^{-1}$ ) as main factors.

\begin{tabular}{|c|c|c|c|}
\hline Especie & Factor & Nivel & Edad (años) \\
\hline \multirow{7}{*}{$\begin{array}{l}\text { N. dombeyi } \\
(\mathrm{n}=179)\end{array}$} & \multirow{3}{*}{ A: Cobertura } & Baja & 5,94 a \\
\hline & & Alta & $8,69 \mathrm{~b}$ \\
\hline & & $\mathrm{F}(*)$ & $26,63(* * *)$ \\
\hline & \multirow{3}{*}{ B: Área basal } & Baja & $6,67 \mathrm{a}$ \\
\hline & & Alta & $7,96 \mathrm{~b}$ \\
\hline & & $\mathrm{F}(*)$ & $5,82(*)$ \\
\hline & $A \times B$ & $\mathrm{~F}(*)$ & 0,00 (ns) \\
\hline \multirow{7}{*}{$\begin{array}{l}\text { N. alpina } \\
(\mathrm{n}=71)\end{array}$} & \multirow{3}{*}{ A: Cobertura } & Baja & $3,04 \mathrm{a}$ \\
\hline & & Alta & $6,70 \mathrm{~b}$ \\
\hline & & $\mathrm{F}(*)$ & $14,54(* * *)$ \\
\hline & \multirow{3}{*}{ B: Área basal } & Baja & $5,41 \mathrm{a}$ \\
\hline & & Alta & 4,33 a \\
\hline & & $\mathrm{F}(*)$ & 1,27 (ns) \\
\hline & $A \times B$ & $\mathrm{~F}(*)$ & 0,60 (ns) \\
\hline \multirow{7}{*}{$\begin{array}{c}N . \text { obliqua } \\
(\mathrm{n}=158)\end{array}$} & \multirow{3}{*}{ A: Cobertura } & Baja & 5,66 a \\
\hline & & Alta & $8,41 \mathrm{~b}$ \\
\hline & & $\mathrm{F}(*)$ & $15,70(* * *)$ \\
\hline & \multirow{3}{*}{ B: Área basal } & Baja & $7,36 \mathrm{a}$ \\
\hline & & Alta & $6.71 \mathrm{a}$ \\
\hline & & $\mathrm{F}(*)$ & $0,86(\mathrm{~ns})$ \\
\hline & $A \times B$ & $\mathrm{~F}(*)$ & 0,77 (ns) \\
\hline
\end{tabular}



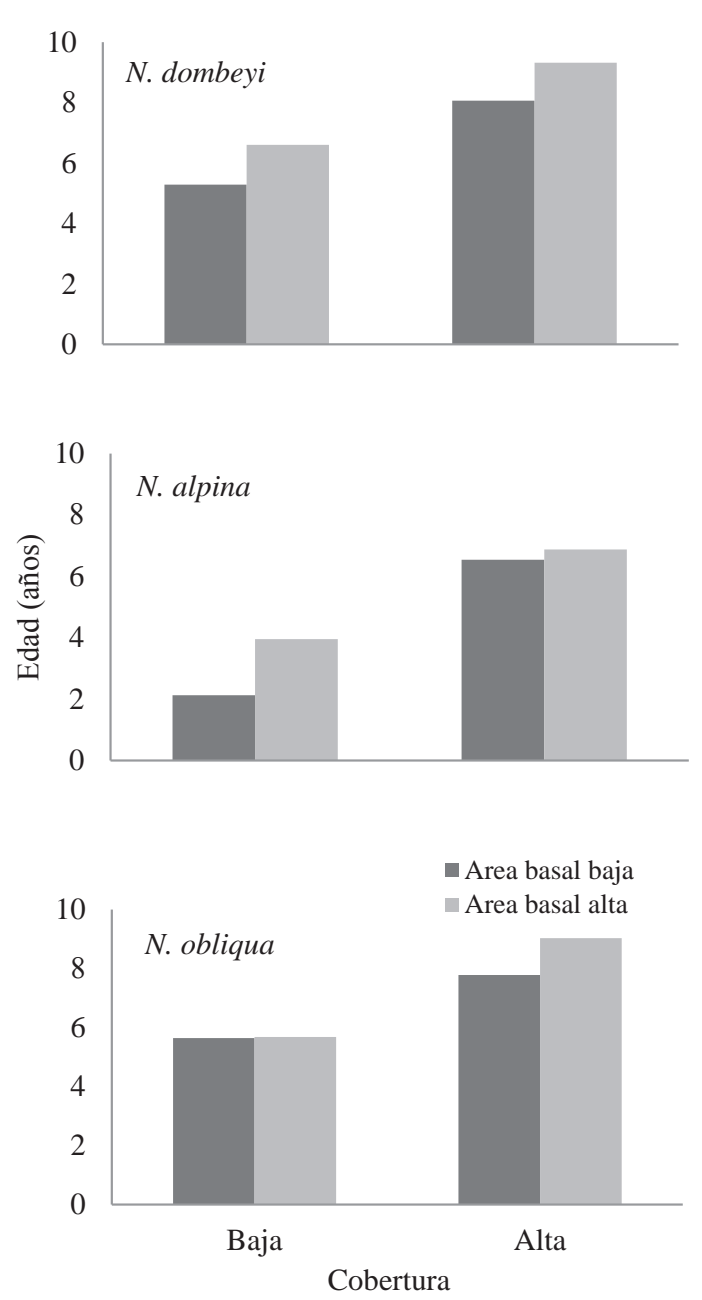

Figura 4. Edad promedio de los renovales con relación al nivel de cobertura y de área basal de árboles adultos de Nothofagus spp. (ANDEVA multifactorial, $P<0,05$ ).

Average age for two levels of canopy tree and basal area of adult trees (Multiple-way ANOVA).

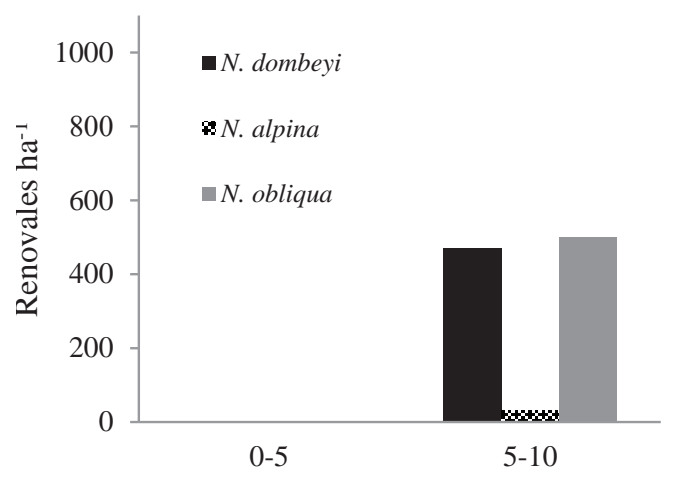

(A) Años luego de la corta

\section{DISCUSIÓN}

Previamente a la implementación del aclareo sucesivo, el bosque estaba representado por un fustal-oquedal cuyos árboles adultos y renovales estaban compuestos principalmente por $N$. alpina. Sin embargo, luego de la corta la regeneración estuvo conformada principalmente por $N$. dombeyi y $N$. obliqua. Estos renovales fueron los de mayor tamaño, mientras que los de $N$. alpina presentaron la menor edad y se localizaron principalmente en sitios con bajas coberturas de dosel. En los sitios con cobertura de dosel mayor, el reclutamiento de las tres especies se inició antes en comparación con el de los sitios más abiertos. La abertura del dosel arbóreo determinó la distribución espacial agrupada de la regeneración en las tres especies, probablemente asociada al aumento de la intensidad y calidad de la luz y al temperamento heliófilo del género Nothofagus. El valor mayor de $\mathrm{I}_{\delta}$ calculado para $N$. dombeyi sería una consecuencia de su mayor densidad.

La mayor tolerancia a la sombra de N. alpina, basada en la comparativa menor tasa de respiración oscura y punto de compensación de luz, habría permitido su establecimiento debajo de las altas coberturas de dosel que caracterizaban al bosque antes de la implementación de las cortas reproductoras $^{5}$ (Pollmann y Veblen 2004, Dezzotti 2008). Esta regeneración avanzada habría sido luego liberada con la creación de aperturas en el dosel, teniendo en cuenta que las de pequeña escala, causadas por la caída de árboles individuales o como consecuencia de cortas selectivas, tienen profundos efectos positivos sobre la regeneración de esta especie (Pollmann 2002, Pollmann 2004).

Luego de la corta diseminatoria, $N$. dombeyi y $N$. obliqua exhibieron un mayor reclutamiento, probablemente asociado a los cambios en el régimen de luz. Estas espe-

Ver nota 2. 
cies poseen una mayor exigencia lumínica que $N$. alpina (Weinberger y Ramírez 1999, 2001, Dezzotti et al. 2003, 2008) y, en consecuencia, la densidad de renovales de estas especies más intolerantes a la sombra es generalmente mayor en sitios recientemente perturbados (Pollmann y Veblen 2004). La apertura del dosel provocada por las intervenciones silviculturales de mayor escala brindan la oportunidad a $N$. dombeyi y $N$. obliqua de colonizar los espacios en los que se han liberado recursos. En áreas similares a la de este estudio, luego de 14 años de ocurrido el aprovechamiento la regeneración de $N$. dombeyi representó el $90 \%$ (Chauchard et al. 1998).

Las acciones de manejo forestal deben tener en cuenta no sólo la cantidad sino también la posibilidad de supervivencia de los renovales que colonizan las áreas abiertas (Chauchard et al. 2001). En los sitios con menor apertura de dosel, los renovales presentaron una mayor edad y, por lo tanto, una mayor cantidad de plantas establecidas. En consecuencia, las condiciones y recursos necesarios para la colonización, principalmente asociados a la luz y humedad, se manifestaron antes en comparación con sitios más abiertos. Estas plantas superaron los $4 \mathrm{~m}$ de altura y por lo tanto presentan una alta probabilidad de conformar el dosel superior. Nothofagus dombeyi y N. obliqua fueron las especies con mayor densidad de renovales de mayor tamaño en la regeneración post-aprovechamiento, corroborándose nuevamente su comportamiento más heliófilo en comparación con $N$. alpina. Este fenómeno fue más marcado en áreas con baja cobertura, donde los renovales de $N$. alpina presentaron la menor edad debido a la gran cantidad de plántulas que se instalaron luego de 15 años de realizadas las prácticas silviculturales.

El área basal de los árboles adultos no mostró relación con la densidad ni con la proporción de especies de renovales. La independencia entre la composición específica de adultos y renovales de Nothofagus spp. en claros formados por cortas de selección en bosquete fue citada previamente (Dezzotti 2003). Además, los renovales de $N$. dombeyi se instalaron antes cuando la cantidad de árboles adultos de esta especie fue mayor. Cabe destacar que los individuos adultos de $N$. dombeyi fueron los de menor densidad con respecto al total de árboles padre, mientras que la regeneración de esta especie fue una de las mayoritarias. $N$. dombeyi produce una cantidad significativamente mayor de semillas por individuo con un tamaño y peso significativamente menor en comparación con $N$. obliqua y $N$. nervosa (Burschel et al. 1976), lo que conduciría a una mayor capacidad de dispersión anemócora (Dezzotti 2003).

Considerando que $N$. dombeyi, $N$. alpina y $N$. obliqua poseen distintos requerimientos lumínicos, es necesario diseñar prácticas silviculturales que combinen adecuadamente la protección de la canopia y la disponibilidad de luz para lograr el establecimiento y desarrollo de las plántulas en las proporciones originales (Donoso et al. 2013). Sin embargo, se debería verificar el grado de influencia de las distintas variables ambientales que son modificadas por la intervención silviculturales y que podrían afectar el establecimiento de los renovales. Además, es necesario estudiar el crecimiento y la sobrevivencia de la regeneración para conocer la composición y estructura del rodal adulto. En este sentido, el desbalance en la proporción de especies podría ser compensado por la capacidad de rebrote de $N$. alpina y $N$. obliqua, aunque el crecimiento y la xilotecnología de estos árboles no se ha estudiado aún.

El mantenimiento de la composición específica de árboles adultos del bosque original, que se lleva a cabo en la actualidad en el aclareo sucesivo, no asegura la misma proporción de especies en la regeneración, al menos durante la etapa post-intervención temprana. La retención de una cobertura superior a la prescripta no sólo acortaría el periodo de establecimiento de los renovales de Nothofagus en general, sino también favorecería la instalación de $N$. alpina en particular. Además es vital la protección de la regeneración avanzada, particularmente dominada por $N$. alpina, ya que exhibirá mayores ventajas en el crecimiento al liberarse mediante la creación de huecos en el dosel. La disminución eventual de los beneficios económicos, asociados al mantenimiento de una mayor cobertura, podría compensarse a través del manejo de la regeneración instalada post-aprovechamiento, promoviendo la liberación de los individuos con mayor capacidad de crecimiento y el establecimiento de la especie económicamente más valiosa N. alpina.

\section{AGRADECIMIENTOS}

Los autores agradecen la colaboración del Departamento Forestal del Parque Nacional Lanín, de la cátedra de Ordenación Forestal de la Universidad Nacional del Comahue y del Grupo de Genética Ecológica y Mejoramiento Forestal del INTA Bariloche. La investigación fue financiada por el Instituto Nacional de Tecnología Agropecuaria (Proyecto PNFOR 044001 "Domesticación de especies forestales nativas") y por la Universidad Nacional del Comahue (Proyecto 04/S016 "Ecología y manejo del bosque mixto de Nothofagus: un avance hacia la conservación"). Finalmente, un particular agradecimiento a los evaluadores, cuyos comentarios y recomendaciones mejoraron sustancialmente la calidad del trabajo.

\section{REFERENCIAS}

Barik S, R Tripathi, H Pandey, P Rao. 1996. Tree regeneration in a subtropical humid forest: effect of cultural disturbance on seed production, dispersal and germination. Journal of Applied Ecology 33: 1551-1560.

Burschel P, C Gallegos, O Martínez, W Moll. 1976. Composición y dinámica regenerativa de un bosque virgen mixto de raulí y coigüe. Bosque 1: 55-74.

Chauchard L, L Maresca, M González Peñalba. 2001. Método para evaluar el estado regenerativo de un rodal y su aplicación al manejo del bosque mixto de Nothofagus. Quebracho 9: 29-42. 
Chauchard L y R Sbrancia. 2003. Modelos de crecimiento diamétrico para Nothofagus obliqua. Bosque 24(3): 3-16.

Dezzotti A. 2008. Acumulación y asignación de biomasa en renovales de tres especies de Nothofagus en los extremos de un gradiente de luz en claros de un bosque subantártico. Investigación Agraria: Sistemas y Recursos Forestales 17(1): 18-30.

Dezzotti A, R Sbrancia, M Rodríguez-Arias, D Roat, A Parisi. 2003. Regeneración de un bosque mixto de Nothofagus (Nothofagaceae) después de una corta selectiva. Revista Chilena de Historia Natural 76: 591-602.

Donoso P, D Soto, R Coopman, S Rodríguez-Bertos. 2013. Early performance of planted Nothofagus dombeyi and Nothofagus alpina in response to light availability and gap size in a high-graded forest in the south-central Andes of Chile. Bosque 34(1): 23-32.

Ferrer J, J Irizarri, J Mendía. 1991. Estudio regional de suelos de la provincia de Neuquén. Consejo Federal de Inversiones- Consejo de Planificación y Acción para el Desarrollo de Neuquén 1: 1-159.

Finkeldey R y M Ziehe. 2004. Genetic implications of silvicultural regimes. Forest Ecology and Management 197: 231-244.

Frazer G, C Canham, K Lertzman. 2000. Gap Light Analyserversion 2.0. Bulletin of the Ecological Society of America 81: 191-197.

Grosse H. 1988. Crecimiento de plantaciones de raulí y roble bajo dosel en dependencia del grado de luminosidad y fertilización. Ciencia e Investigación Forestal 2(5): 13-30.

Martínez Pastur G, P Peri, C Fernández, G Staffieri, M Lencinas. 2002. Changes in understory species diversity during the Nothofagus pumilio forest management cycle. Journal of Forest Research 7(3): 165-174.

Morisita M. 1959. Measuring the dispersion of individuals and analysis of the distributional patterns. In Memory Faculty of
Science Kyushu University. Fukukoa, Japón. Serie E (Biology) 2. p. 215-235.

Pollmann W. 2002. Effects of natural disturbance and selective logging on Nothofagus forests in south-central Chile. Journal of Biogeography 29: 955-970.

Pollmann W. 2004. Regeneration dynamics and life history differences in southern Chilean Nothofagus forests: a synthesis. Plant Ecology 174: 353-369.

Pollmann W y T Veblen. 2004. Nothofagus regeneration dynamics in south-central Chile: a test of a general model. Ecological Monographs 74(4): 615-634.

Promis A, J Caledentey, M Ibarra. 2010. Microclima en el interior de un bosque de Nothofagus pumilio y el efecto de una corta de regeneración. Bosque 31(2): 129-139.

Ramírez C, C San Martín, A Oyarzún, H Figueroa. 1997. Morpho-ecological study of the South American species of the genus Nothofagus. Plant Ecology 130: 101-109.

Read J y R Hill. 1985. Photosynthetic responses to light of Australian and Chilean species of Nothofagus and their relevance to the rain forest dynamics. New Phytologist 101: 731-743.

Smith D, B Larson, M Kelty y P Ashton. 1997. The practice of silviculture: applied forest ecology. New York, USA. John Wiley \& Sons. 537 p.

Veblen T, C Donoso, T Kitzberger, A Rebertus. 1996. Ecology of southern Chilean and Argentinian Nothofagus forests. In Veblen T, R Hill S, J Read eds. The ecology and biogeography of Nothofagus forests. Yale New Haven, CT, USA. University Press. p. 293-353.

Weinberger P y C Ramírez. 1999. Sinecología de la regeneración natural del raulí (Nothofagus alpina). Revista Chilena de Historia Natural 72: 337-351.

Weinberger P y C Ramírez. 2001. Microclima y regeneración natural de raulí, roble y coigüe (Nothofagus alpina, N. obliqua y N. dombeyi). Bosque 22(1): 11-26. 